\title{
DYNAMIC MEASUREMENT OF FOAM-SIZED YARN PROPERTIES FROM YARN SEQUENCE IMAGES
}

\author{
Bo Zhu, Zhongjian Li, Xinwei Cao, Jianli Liu, Weidong Gao*
}

Jiangsu Engineering Technology Research Center for Functional Textiles, Jiangnan University, Wuxi, China, 214122

Corresponding author E-mail: gaowd3@163.com

\section{Abstract:}

Unlike the normal sizing method, the foam sizing had been proven to be a low-add-on technology. To investigate the effect of foam sizing, film thickness, sized-yarn evenness, and size penetration rate were necessary to evaluate the performances of foam-sized yarns. However, the conventional image analysis of sized-yarn cross sections primarily relied on artificial testing with a low efficiency. This paper proposed a novel dynamic method to measure the sized-yarn properties including film thickness, sized-yarn evenness, and size penetration rate based on yarn sequence images captured from a moving yarn. A method of dynamic threshold module was adopted to obtain threshold for segmenting yarns in the sequence images. K-means clustering algorithm was applied to segment pixels of the images into yarn and background. To further remove burrs and noise in the images, two judgment templates were carried out to extract the information of yarn core. The film thickness, sized-yarn evenness, and size penetration rate were measured based on the yarn core of each frame in sequence images. In order to compare with the experimental results of the dynamic method, the yarn properties of the same samples were tested by static and artificial testing. Results revealed that the proposed method could efficiently and accurately detect the film thickness, sized-yarn evenness, and size penetration rate.

\section{Keywords:}

Foam sizing; Yarn sequence image; K-means clustering; Yarn diameter; Yarn evenness; Size penetration rate

\section{Introduction}

Foam technology, such as foam dyeing [1-4] and foam finishing [5-7], developed more rapidly in textile. Foam sizing was proved to be an energy-saving emission reduction technology, new technology of using bubbles as the medium for applying size to yarns. The objectives of yarn sizing were to improve the yarn wear resistance and decrease the yarn hairiness in order to guarantee the weaving process to carry on smoothly. The principle of foam sizing was that most of the size coated on the surface of yarns and a small amount of size penetrated into the yarns. Owing to using foams as media instead of size liquor, the size pick-up of foam-sized yarns were lower, the burden of yarn drying was significantly reduced and desizing became more easier compared with the conventional sizing. The excellent energy-saving and pro-environmental performances made the technology of foam sizing to get the favors of cotton textile enterprise. Namboodri [8], Trauer and Vialon [9, 10], Vernekar [11], Shah and Gandhi [12], and Berk [13] studied the effects of foam sizing during the 1980 s and 1990s and indicated the advantages of foam sizing. This technology was halted to investigate because of severing contemporary constraints of mechanical technology. Until in 2014, Weidong Gao $[14,15]$ in the Jiangnan University broke through the limitations and developed foam sizing for industrial application and studied sizing effects of foam-sizing and warp pre-wetting combined process.

Breaking elongation, abrasion resistance, hairiness index, film thickness, sized-yarn evenness, and size penetration rate were considered as the significant properties to evaluate the sizing quality. Among these properties, breaking elongation, abrasion resistance, and hairiness index were conventionally measured by yarn strength tester, yarn abrasion tester, and Uster tester, respectively. In the paper, film thickness, sized-yarn evenness, and size penetration rate that described the coating and penetration of size were defined as detail properties of sized yarns. Conventionally, the three detail properties were tested by using the method of yarn cross-section test. This testing method was a kind of static visual inspection that includes steps such as section preparation, microscopic image acquisition, and the feature extraction of yarn cross section. Among these steps, the cross sections were prepared by artificial and accessed randomly, which made the testing results inaccurate. Thus, this manual method was barely used because of the long testing time, complex testing procedures, and high test cost. Nowadays, with the widely studied foam sizing technology, it was necessary to investigate a faster and more effective method to explain the mechanism of the new sizing technology.

Currently, image analysis seemed to be a trend in developing methods to measure the yarn property [16-24]. It was generally believed that visual inspection was the best approach to evaluate the detail properties of the sized yarn more efficiently [25]. This paper proposed a dynamic measurement for the sized-yarn detail properties from yarn sequence images with no damage to the yarn structure. The yarn diameter was obtained by measuring the width between yarn edges to calculate the film thickness of sized yarn. The sized-yarn evenness 
was equivalent to the evenness of sized-yarn diameter. Size penetration rate was the proportion of the penetration of size volume and yarn volume. Obviously, the measurement of the detail properties of the sized yarn using image analysis was still a relevant topic.

In this research, the successive yarn images were dynamically acquired by an integrated image capturing and processing system. A dynamic threshold module was applied to obtain global threshold values for segmenting yarn sequence images. The film thickness, sized-yarn evenness, and size penetration rate were measured based on the yarn core of each frame in the sequence images. The results of sized-yarn detail properties were tested by dynamic method and compared to the detection results by static cross-section testing and artificial weight testing.

\section{Experimental details}

\subsection{Sample Preparation}

In this experiment, four kinds of $100 \%$ cotton single greige yarnsas shown in Table 1, which were purchased from Yancheng Yueda Cotton Spinning Co., Ltd, were selected to evaluate the yarns properties. On the basis of the previous studies, oxidized starch and polyacrylic acid blended in proportion of 50:50 and $15 \%$ of size concentration were used to size the samples of greige yarns by GA168 foam-sizing machine. Greige yarn of 10 $m$ in length and sized yarn were tested by the proposed method for each yarn sample. The experiments were carried out in triplicate; averages and standard deviations were obtained. All of the yarn samples were stored under a relative humidity of 65 $\pm 5 \%$ at $25 \pm 5^{\circ} \mathrm{C}$.

\subsection{Yarn image acquisition device}

The yarn images of $10 \mathrm{~m}$ length were dynamically and continuously captured and automatically processed by the yarn image acquisition device as displayed in Figure 1.

The sealed imaging box (1) was used as the image acquisition platform in the experiment (Figure 2). The sample images were captured in the sealed box without the interference of lighting variation. A special light source was adopted to set up in the sealed box. In addition, the combination of a yarn winding mechanism, a servo motor, and a tension controller was applied to draw the yarn and to stabilize the tension of the moving yarn. Snapshot photographs were successively taken along the yarn by an area-scan charge-coupled device (CCD) camera.

In order to process rapidly, the yarn images of four samples $(\# 1, \# 2, \# 3$, and \#4) and sized-yarn images (\#1', \#2', \#3', and $\# 4^{\prime}$ ) were captured at the size of 600 pixels $\times 768$ pixels through cropping the images at a suitable width, which were shown in Figure 3. A pixel occupied $5.21 \mu \mathrm{m}$ in the image, and the actual length of yarn in the captured image was approximately $4 \mathrm{~mm}$. The yarn running speed was precisely set at $10 \mathrm{~m} / \mathrm{min}$.

Table 1. Four kinds of yarn samples.

\begin{tabular}{|c|c|c|c|}
\hline Sample number & Yarn tex & Spinning method & Material \\
\hline$\# 1$ & 19.4 & Ring spinning & Cotton \\
\hline$\# 2$ & 14.6 & Ring spinning & Cotton \\
\hline$\# 3$ & 11.7 & Ring spinning & Cotton \\
\hline$\# 4$ & 9.7 & Ring spinning & Cotton \\
\hline
\end{tabular}

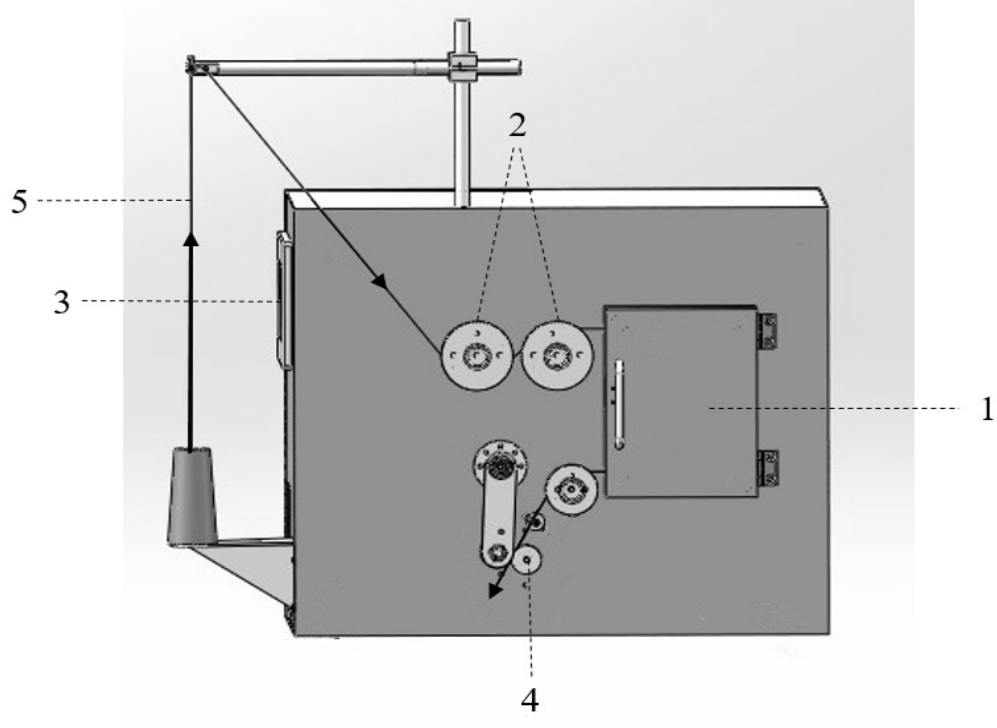

Figure 1. The integrated structure of yarn image acquisition device: (1) sealed imaging box; (2) yarn tension control panel; (3) touch screen; (4) output rollers with servo motor yarn; (5) yarn. 


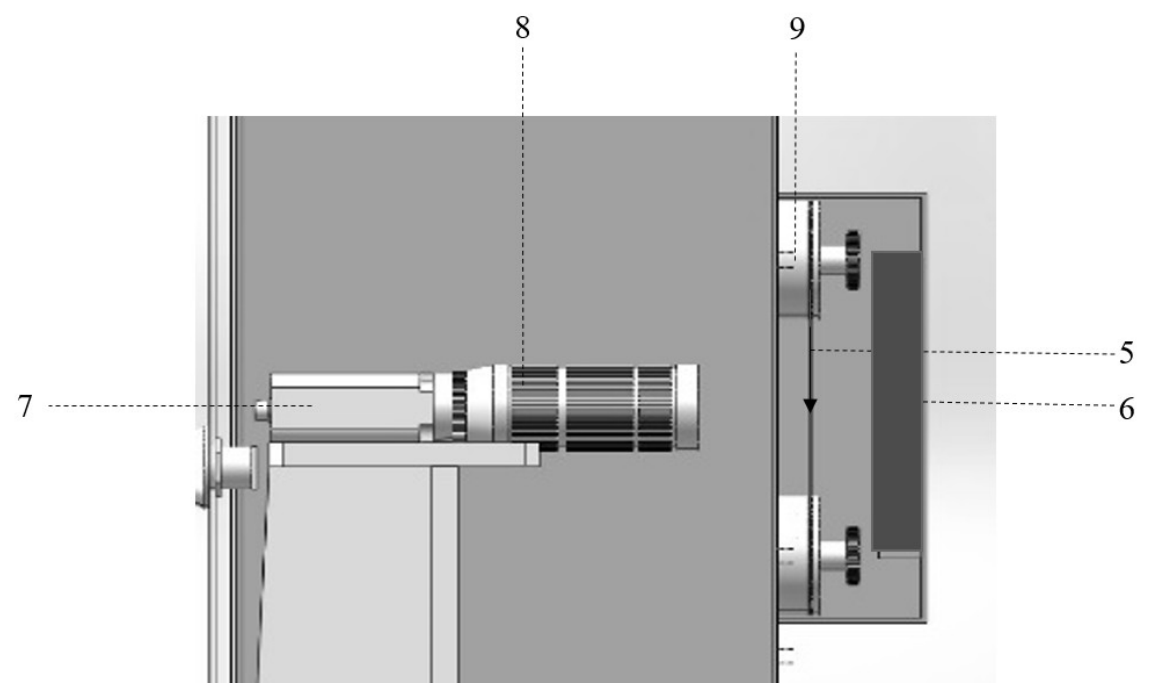

Figure 2. The integrated structure of sealed imaging box: (5) yarn; (6) light source; (7) area-scan CCD camera sensor; (8) camera lens; (9) output rollers with servo motor.

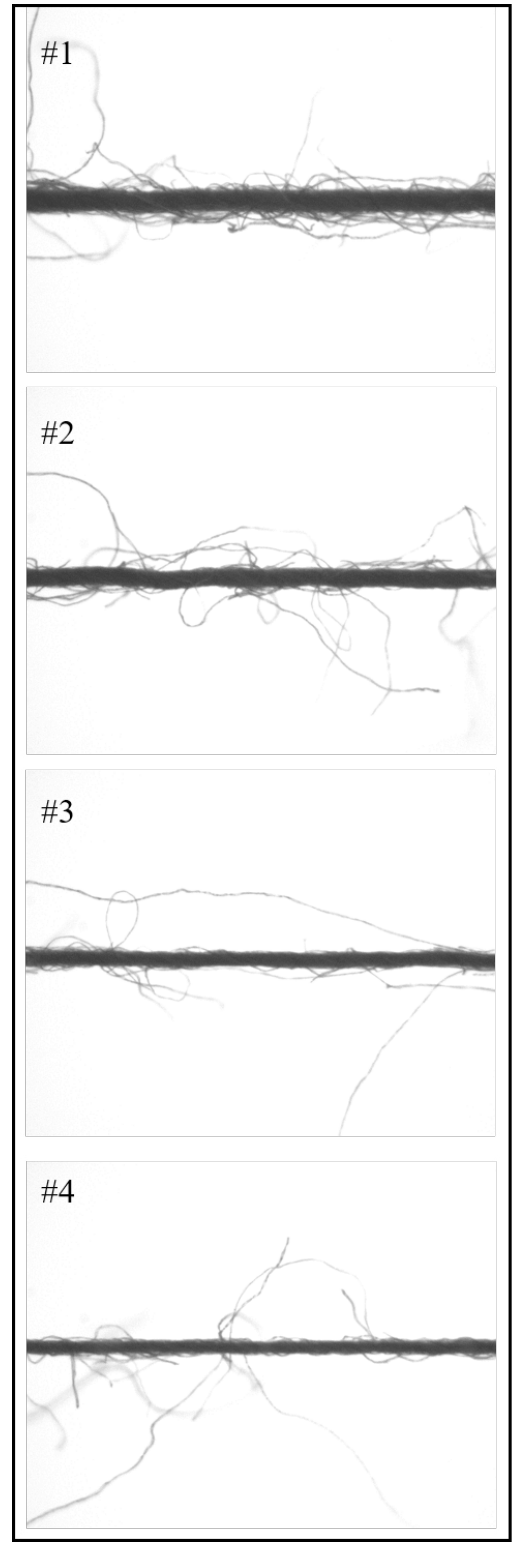

(a)

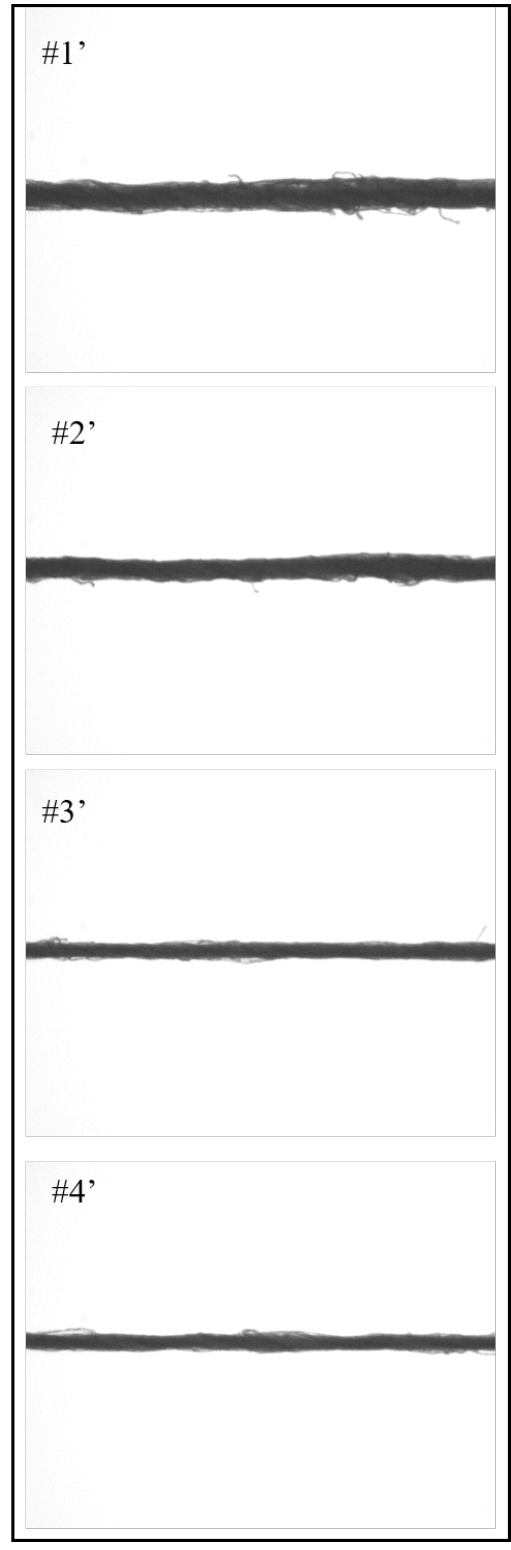

(b)

Figure 3. Four kinds of yarn images: (a) greige yarn images; (b) sized-yarn images 


\section{$\underline{2.3 \text { Image processing }}$}

The determination of threshold values for segmenting the yarn images automatically was a critical part of measuring yarn diameter and directly impacted the precise of yarn properties by image processing. Manual setting or grayscale threshold methods, such as Ostu method [26], gray-level histogram method [27], and maximum-entropy automatic threshold [28], were the general methods to determine the threshold values of images. Owing to the artificial operation and time consumption, these methods were unsatisfied to process the yarn sequence images automatically in real time. To realize the saving of labor and time, dynamic threshold was proposed in this article to obtain the threshold values. This dynamic method was a kind of faster algorithm and can faster and automatically determine the threshold values of the sequence images without depending on manual operation.

In this research, clustering algorithm was used to decide the segmentation threshold. The information of clustering center of the former frame can be used to the next image because the gray property of the yarn sequence images obtained from the device was similar. The clustering center of the former frame was recorded as the initial clustering center of the next frame to reduce the time of calculation. According to the operational time studied from the earlier research [29], three clustering methods of K-means clustering algorithm were selected to segment the yarn image. The local average intensity and entropy of image were used as the classification vector that was calculated in a $3 \times 3$ template as shown in the following equations, and the 8-neighborhood points were applied to obtain the vectors for a given pixel $P_{11}$ [29].

$$
\begin{aligned}
& A\left(P_{11}\right)=\frac{1}{9} \sum_{i=0}^{2} \sum_{j=0}^{2} P_{i j} \\
& E\left(P_{11}\right)=\sum_{i=0}^{2} \sum_{j=0}^{2} \rho_{i j} \log \left(\rho_{i j}\right) \\
& \rho_{i j}=P_{i j} / \sum_{i=0}^{2} \sum_{j=0}^{2} P_{i j}
\end{aligned}
$$

where $A\left(P_{11}\right), E\left(P_{11}\right)$, and $\rho_{i j}$, respectively, represent the average intensity value, the entropy value, and the proportion of every points in $3 \times 3$ template. $P_{i j}$ is the grayscale value of the 8-neighborhood points of $P_{11}$. The running time and the determined threshold of the four greige yarn samples by $\mathrm{K}$-means algorithm based on the two vectors were exhibited in Table 2.

Table 2. The comparison of three clustering methods of K-means family.

\begin{tabular}{|c|c|c|}
\hline Sample number & $\begin{array}{c}\text { Running Time } \\
\mathbf{( s )}\end{array}$ & $\begin{array}{c}\text { Determined } \\
\text { threshold }\end{array}$ \\
\hline$\# 1$ & 0.094 & 133 \\
\hline$\# 2$ & 0.085 & 142 \\
\hline$\# 3$ & 0.102 & 126 \\
\hline$\# 4$ & 0.114 & 150 \\
\hline
\end{tabular}

As shown in Table 2, the threshold values of different sample images were determined within about $0.1 \mathrm{~s}$, which indicate that K-means clustering algorithm was capable of segmenting the threshold values to obtain the binary yarn images and significantly saved the time of computation.

After foam sizing, the harmful hairiness (more than $3 \mathrm{~mm}$ ) of sized yarns were significantly reduced and some burrs still remained on the yarn surface. To measure the yarn diameter accurately, the burrs and noise in the yarn images should be removed. Two judge templates were adopted to remove the pixels of burrs and some noise after threshold processing. It was observed that the maximum size of yarn hairiness captured by the image acquisition device was about 5 pixels $\times 2$ pixels in the binary images. The two 5 pixels $\times 2$ pixels of judge templates were used to scan every dot of binary yarn image. The white pixels with the value of 1 and the black pixels with the value of 0 , respectively, represented the yarn and the background dots. The white pixel was set as the object pixel. All the values were summed together in the two templates when the current point was white. If the sum of both the values was less than 8 , then the value of current point was set to 0 as background information; otherwise the value remained 1 as a yarn dot. The calculations were executed several times until all of the burrs and noise points were eliminated. Taking the \#2 yarn as example, the processing results of greige yarn images were shown in Figure 4.

Figure 4 showed that the interference information of binary yarn image was completely removed and the yarn core information was perfectly preserved by the above method. The judge templates successfully and efficiently eliminated the burrs, unrelated points, and small areas in yarn binary image.

\section{Data analysis and discussion}

\subsection{Statistical parameters of varn properties}

\subsubsection{Film thickness}

The film was a covering coating layer around the yarn surface that protected the yarn from mechanical friction in the weaving process. Film thickness was the difference in diameter between the greige yarn and the sized yarns. Although the thicker film enhanced the abrasion resistance of the sized yarns, it tended to increase the yarn diameter, which was not conducive to the high-speed weaving of superfine count high-density fabric. While the less coating size made the film thinner, it resulted in pilling and increased broken ends during the weaving process. The methods used for calculating film thickness were shown as following equations:

$$
\begin{aligned}
& r_{i}=\left(p_{j}-q_{j}\right) \times 5.21 \\
& \bar{r}=\frac{\sum_{i=0}^{W} r_{i}}{W} \\
& R_{i}=\left(m_{j}-n_{j}\right) \times 5.21
\end{aligned}
$$


a)
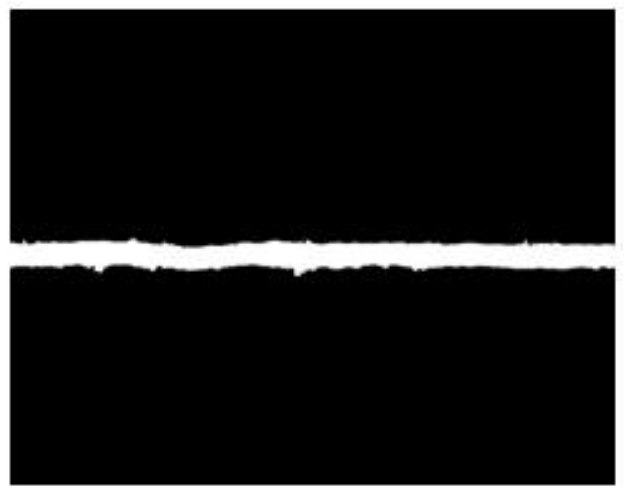

b)
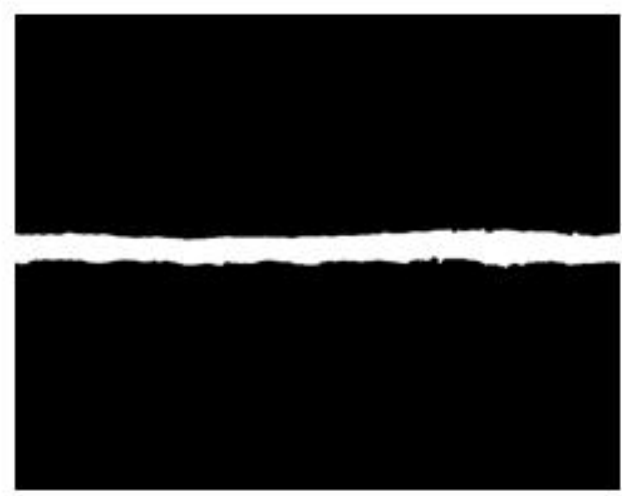

Figure 4. The images after processing by two judge template: (a) greige yarn image (\#2), (b) sized-yarn image (\#2’)

$$
\begin{aligned}
& \bar{R}=\frac{\sum_{i=0}^{W} R_{i}}{W} \\
& R_{0}=\frac{\bar{R}-\bar{r}}{2}
\end{aligned}
$$

where $\bar{r}$ and $\bar{R}$ represented the average diameter of greige yarn and sized yarn, respectively. The size of image was $i \times j$. $W$ denoted the total number of columns of the image. $r_{i}$ and $R_{i}$ denoted the diameter at column i. $p_{j}$ and $q_{j}$ were the first and the last white pixel, respectively, at each column in the greige yarn image. $m_{j}$ and $n_{j}$ were the first and the last white pixel, respectively, at each column in the sized-yarn image. $p_{j}-q_{j}$ and $m_{j}-n_{j}$ were the sum of white pixels. $\ddot{\mathrm{A}} R_{i}$ was the film thickness at column $i . R_{0}$ represented the average value of film thickness. One pixel in the horizontal direction occupied $5.21 \mu \mathrm{m}$ in the image. According to equations (4)-(8), the average value was calculated as equation (9):

$$
R_{0}=\frac{\frac{\sum_{i=0}^{W} R_{i}}{W}-\frac{\sum_{i=0}^{W} r_{i}}{W}}{2}=\frac{\sum_{i=0}^{W}\left(m_{j}-n_{j}-p_{j}{ }_{j}\right) \times 2.605}{W}
$$

The diameter of greige yarns and sized yarns and film thickness of four kinds of yarn, which listed in Table 1, were measured using equation (9). The measurement results were shown in Table 3.

Table 3. The yarn diameter and film thickness
In Table 3, the film thickness of each sample was calculated by subtracting the diameter of sized yarn and greige yarn. A thin film was successfully formed on the yarn surface after foam sizing, which made the majority of harmful hairiness sticking to the yarn surface and increased the break strength. The film also resisted the yarn abrasion during the weaving process. The thickness of film could be adjusted by changing the parameters of sizing process, such as foaming ratio, squeezing pressure, and the density of size liquor.

\subsubsection{Sized-yarn evenness}

The yarn property of film thickness just indicated the formation of a single layer around the yarn surface after foam sizing. The foam-sized yarns should be evenness that was benefit to weave smoothly. In additional to the overall analysis and measurement of sized-yarn evenness, the evenness of yarn diameter was also an important index to evaluate the quality of foam sizing. The yarn unevenness resulted in a high possibility of yarn breakage in weaving process. Variation coefficient of the yarn diameter calculated by equations (10) and (11) was the ratio between the standard deviation and the average value, which dictated the discrete degree of yarn diameter in unit length. The smaller value of coefficient of variation indicated that the sized yarn was more even.

\begin{tabular}{|c|c|c|c|}
\hline \multirow{2}{*}{ Sample number } & \multicolumn{2}{|c|}{ Diameter } & \multirow{2}{*}{ Film thickness $(\boldsymbol{\mu m})$} \\
\cline { 2 - 4 } & Greige yarn $(\boldsymbol{\mu m})$ & Sized yarn $(\boldsymbol{\mu m})$ & 3.39 \\
\hline$\# 1$ & 167.11 & 173.89 & 2.98 \\
\hline$\# 2$ & 134.95 & 140.91 & 3.06 \\
\hline$\# 3$ & 108.45 & 114.57 & 3.24 \\
\hline
\end{tabular}


Table 4. The coefficient of variation of yarns

\begin{tabular}{|c|c|c|}
\hline \multirow{2}{*}{ Sample number } & \multicolumn{2}{|c|}{ Coefficient of variation (\%) } \\
\cline { 2 - 3 } & Greige yarn & 6.32 \\
\hline$\# 1$ & 12.47 & 5.06 \\
\hline$\# 2$ & 14.59 & 5.43 \\
\hline$\# 3$ & 17.83 & 4.69 \\
\hline$\# 4$ & 15.42 & \multicolumn{2}{|c|}{ Sized yarn } \\
\hline
\end{tabular}

$$
\begin{aligned}
& C V 1=\frac{\sqrt{\frac{1}{W} \sum\left(r_{i}-\bar{r}\right)^{2}}}{\bar{r}} \times 100 \% \\
& C V 2=\frac{\sqrt{\frac{1}{W} \sum\left(R_{i}-\bar{R}\right)^{2}}}{\bar{R}} \times 100 \%
\end{aligned}
$$

where CV1 and CV2, respectively, represented the evenness of greige yarn and sized yarn. The coefficient of variation of four yarn samples, which was listed in Table 1, was measured by the above equations. The measurement results were depicted in Table 4.

The coefficient of variation of sized yarns shown in Table 4 was significantly declined after foam sizing compared to that of greige yarns, which implied the evenness of sized yarns became better. The yarn unevenness after foam sizing was improved so as to decrease the yarn breakage during the process of weaving. In the sizing process, it was worthy nothing that the yarn tension and the speed of slasher should be controlled in a stable and proper parameter to keep the yarn tension uniform.

\subsubsection{Size penetration rate}

Through the pressure by the squeezing roller, the bubbles broke and released the containing size liquor. The major part of size coated on the surface of yarn and formed a thin film after oven drying, and a certain amount of size liquor penetrated into the yarn. Size penetration rate was a critical detail property to enhance the break strength and abrasion resistance of the yarns. The excessive penetration rate made the fabric rough when the desizing process was incomplete to leave the residual size in the fabric. Size penetration rate is equal to the ratio between the volume of the size penetrated into the yarn and the volume of greige yarn.

$$
\begin{aligned}
& m_{2}=m_{1}-m_{0} \\
& m_{3}=\rho\left(V_{1}-V_{0}\right)=h \rho \pi \sum_{i=0}^{W}\left(\frac{R_{i}^{2}}{4}-\frac{r_{i}^{2}}{4}\right) \times 10^{-8}=1.3025 \times 10^{-12} \rho \pi \sum_{i=0}^{W}\left(R_{i}{ }^{2}-r_{i}^{2}\right) \\
& m_{4}=m_{2}-m_{3} \\
& P R=\frac{V_{p}}{V_{0}}=\frac{m_{4} / \rho}{h \rho \pi \sum_{i=0}^{W}\left(\frac{r_{i}^{2}}{4} \times 10^{-8}\right)}=\frac{m_{1}-m_{0}-1.3025 \times 10^{-12} \rho \pi \sum_{i=0}^{N}\left(R_{i}{ }^{2}-r_{i}{ }^{2}\right)}{1.3025 \times 10^{-12} \rho \pi \sum_{i=0}^{W} r_{i}{ }^{2}}
\end{aligned}
$$


The preparation of cross-section slice was one of these key steps that directly affected the image quality of cross-section slice, thus affected the precise of the feature extraction. Many typical methods have already been used to make cross-section slice, such as hand cutting and hand-operated slicer. The slice made by hand cutting was too thick to capture a legible image which quickly became obsolete. By hand-operated slicer, the cross-section slice was produced using paraffin-coated yarn. These manual methods had some disadvantages of operating inconvenience and difficulties in slice preparation. Recently, some researchers used scanning electron microscope (SEM) to observe and analyze the yarn cross-section slice. Owing to the high cost, this method was only eligible to some specific investigation and inappropriate to extensive research. Currently, hardy's thin cross-section device (Y172) was widely used to prepare slice because of the simple and convenient operation. In general, the hardy's thin cross-section method was adopted to measure the coating rate and penetration rate of sized yarn. However, the coating rate of size on the yarn surface barely demonstrated the film thickness and sized-yarn evenness. Thus, this paper tested the film thickness and sizedyarn evenness of cross-section slice image instead of size coating rate to vividly illustrate the quality of foam sizing. As mentioned earlier, the yarns in the experiments were sized by oxidized starch and polyacrylic acid. Owing to the color reaction between iodine and starch, the sized yarn was first processed by potassium iodide. Then the sized yarn was enclosed by wood fiber and sheared by hardy's thin cross-section device to prepare slices. In order to prevent the sized-yarn distorting under the compression of wood fiber, the sized yarn was preprocessed by collodion. The thickness of slice was around 20 $\mu \mathrm{m}$. The cross section was sliced every $0.5 \mathrm{~m}$, and 20 slices were obtained. In the comparison experiments, the images of slices were observed by fiber detecting system (YG002C) and captured by light microscope with a $500 \times$ magnification.

\subsubsection{Static image testing method}

This static testing method included steps such as the preparation of sized-yarn cross-section slice, microscopic image acquisition, and the feature extraction of slice image. According to the image testing method proposed by $\mathrm{Yu}$, the images of cross-section slices were processed via gray, binarization, image denoising, and enhancement. After the image pre-processing, the area of coating and penetration size were extracted and statistically computed to calculate the detail properties after morphological image processing. Taking the yarn of 9.7 tex as example, the film thickness, sized-yarn evenness, and size penetration were calculated based on the images of 20 slices.

\subsubsection{Artificial testing method}

The captured slice image was manually drawn in a tracing paper. The film thickness, sized-yarn evenness, and size penetration were tested by measuring the length and weighting. The results calculated by artificial testing method were more accurate and considered as practical value. The detail properties of 20 slice images were traced and obtained. Table 6 shows the results of the proposed dynamic testing (M1), static image testing (M2), and artificial testing method (M3).

To intuitively compare the accuracy of measurement results of the two image testing methods (M1 and M2) with artificial testing method (M3), the absolute values of the difference were displayed in Figure 5.

As clearly seen from Figure 5, the results of dynamic image testing method M1 were more precise. This was mainly due to the M1 that measured each frame image and calculated each column of the yarn diameter. The proposed dynamic method

Table 6. The results of yarn detail properties

\begin{tabular}{|c|c|c|c|c|c|c|c|c|c|}
\hline \multirow{2}{*}{$\begin{array}{c}\text { Sample } \\
\text { number }\end{array}$} & \multicolumn{3}{|c|}{ Film thickness $(\boldsymbol{\mu m})$} & \multicolumn{3}{c|}{ Yarn evenness (\%) } & \multicolumn{3}{c|}{ Penetration rate (\%) } \\
\cline { 2 - 10 } & $\mathbf{M 1}$ & M2 & M3 & M1 & M2 & M3 & M1 & M2 & M3 \\
\hline$\# 1$ & 3.39 & 3.39 & 3.39 & 6.32 & 6.19 & 6.35 & 18.2 & 20.1 & 18.5 \\
\hline$\# 2$ & 2.98 & 2.98 & 2.98 & 5.06 & 4.87 & 5.02 & 21.3 & 22.4 & 20.9 \\
\hline$\# 3$ & 3.06 & 3.06 & 3.06 & 5.43 & 5.63 & 5.39 & 19.5 & 18.3 & 19.6 \\
\hline$\# 4$ & 3.24 & 3.24 & 3.24 & 4.69 & 4.77 & 4.66 & 22.4 & 22.9 & 20.9 \\
\hline
\end{tabular}

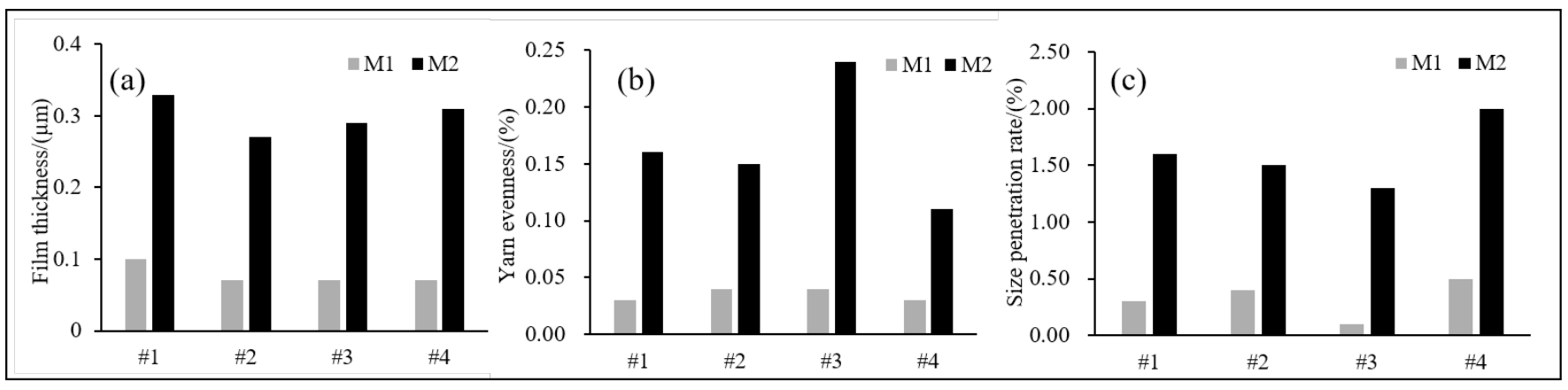

Figure 5. The comparison results of (a) film thickness; (b) yarn evenness; (c) size penetration rate 
significantly saved time for yarn detail properties measurement and improved the accuracy of testing results.

\section{Conclusions}

Yarn detail properties including film thickness, yarn evenness, and size penetration were critical performances to study the mechanism of foam sizing technology. This paper proposed a novel dynamic image processing method to detect yarn detail properties. First, dynamic threshold module was used to acquire threshold for each frame image. Then, the K-means clustering algorithm was applied to segment yarn information of each frame images to avoid the drawbacks of manual settings and reduce the operation time. Two judge templates were used to obtain the yarn core information and remove the burrs and unrelated noises from the binary images. In the experiments, the film thickness, sized-yarn evenness, and size penetration rate were inspected based on the yarn core of each frame in the sequence images. The data obtained from the proposed method were compared with the results tested by static and artificial testing. The comparison results showed that the proposed dynamic method successfully detected the yarn diameter more accurately and efficiently to calculate the film thickness, yarn evenness, and size penetration. In the meantime, the measurement of yarn detail properties had instructional significance for further study on technology parameters of foam sizing.

\section{ACKNOWLEDGMENTS}

The author would like to acknowledge the innovation project of Jiangsu Graduate Education (No. CXZZ13_0749), the scientific research fund project of the Jiangnan University (No. JUDCF13025), the prospective joint research project of Jiangsu Province (No. BY2015019-07), the independent innovation and the transformation of scientific and technological achievements project of Shandong Province (No. 2014CGZH0205), and a project funded by the Priority Academic Program Development of Jiangsu Higher Education Institutions (PAPD).

\section{References}

[1] W. Perkins, R. Walker, Foam Sizing, Textil. Res. J. 9 (1982) 547-554.

[2] K. Lu, X. Zhang, Y. Zhao, Z. Wu, Removal of color from textile dyeing wastewater by foam separation, J. Hazard. Mater. 182 (2010) 928-932.

[3] H. Yu, Y. Wang, Y. Zhong, Z. Mao, S. Tan, Foam properties and application in dyeing cotton fabrics with reactive dyes, Color. Technol. 130 (2014) 266-272.

[4] J.Y. Bae, J.E. Joo, Y.J. Lee, M.S. Han, S.H. Kim, Fabrication of biodegradable polylactide foam for algal bloom control, Fiber. Polym. 3 (2002) 159-168.

[5] W.H. Lee, S.W. Lee, T.J. Kang, K. Chung, J.R. Youn, Processing of polyurethane/polystyrene hybrid foam and numerical simulation, Fiber. Polym. 21 (2015)134-146.

[6] K. Baker, G. Bryant, J. Camp, B. Kelsey, Foam Finishing Technology, Textil. Res. J. 52, (1982) 395-403.
[7] C. Namboodri, M. Duke, Foam Finishing of CottonContaining Textiles, Textil. Res. J. 49 (1979) 156-162.

[8] C. Namboodri, Foam Sizing of Cotton and Blend Yarns: Slashing Trials, Textil. Res. J. 2 (1986) 87-92.

[9] J. Trauter, R. Vialon, Aspects of Foam Sizing, Textiletrieb. 104 (1986) 33-41.

[10] J. Trauter, R. Vialon, State of Foam Sizing, Textil. Asia. 18 (1987) 97.

[11] S. Vernekar, Foam Sizing-Perspectives and Limitations, Manmade Textil. In. India. 35 (1992) 51-52.

[12] D. J. Shah, R. S. Gandhi, Technique of Foam Sizing, J. Ind. Text. 10 (1990) 184-185.

[13] W. Beck, Foam Technology in Yarn Sizing, Textil. Praxis. Int. 623 (1989).

[14] W.D. Gao, X.R. Fan, J.L Liu, J.X. Zhang, L. Du, Technology Study of Foam Sizing, Cot. Textil. Tech. 42 (2014) 1-5.

[15] Y. Lu, J. Zhang, J. Liu, W. Gao, Sizing effects of foamsizing and yarn pre-wetting combined process, J. Textil. Res. 12 (2014) 47-51.

[16]B.G. Xu, C.M. Murrells, X.M. Tao, Automatic measurement and recognition of yarn snarls by digital image and signal processing methods, Text. Res. J. 78 (2008) 439-456.

[17] Z.J. Li, R.R. Pan, W.D. Gao, Formation of digital yarn black board using sequence images Text. Res. J. 2016 (86) 593603.

[18] Y. Guo, Tao X M, Xu B G, Choi K F, Hua $T$ and Wang S Y 2010 A continuous measurement system for yarn structures by an optical method Meas. Sci. Technol. 21 115706-115720

[19] J. Feng, B.G. Xu, X.M. Tao, J. Feng, B.G. Xu, Dynamic measurement and modelling of flexible yarn dynamic behaviour on a moving cylindrical solid structure, Meas. Sci. Technol. 23 (2012) 286-286.

[20] A. Shams Nateri, F. Ebrahimi, N. Sadeghzade, Evaluation of yarn defects by image processing technique, Optik, 125 (2014) 5998-6002.

[21] A. Sengupta, S. Roy, S. Sengupta, Development of a low cost yarn parameterisation unit by image processing, Measurement 59 (2015) 96-109

[22] M. Eldessouki, S. Ibrahim, J. Militky, A dynamic and robust image processing based method for measuring the yarn diameter and its variation, Text. Res. J. 84 (2014) 19481960.

[23] J. Zhang, B. Xin, X. Wu, Density measurement of yarn dyed woven fabrics based on dual-side scanning and the FFT technique, Meas. Sci. Technol. 25 (2014) 1-17.

[24] J. Liu, H. Jiang, X. Liu, Z. Chai, Automatic measurement for dimensional changes of woven fabrics based on texture, Meas. Sci. Technol. 25 (2014) 015602.

[25] P. Zhong, Z. Kang, S. Han, R. Hu, J. Pang, X. Zhang, Evaluation method for yarn diameter unevenness based on image sequence processing, Text. Res. J. 85 (2015) 369-379.

[26] B. Zhu, J. Liu, R. Pan, S. Wang, W. Gao, Fabric seam detection based on wavelet transform and CIELAB color space: A comparison, Optik, 126 (2015) 5650-5655.

[27] S. Behtaj, S. Sadri, H. Tavanai, Objective yarn bulk measurement through image analysis, J. Text. Inst. 102 (2011) 1094-1100. 
AUTEX Research Journal, Vol. 18, № 3, September 2018, DOI: 10.1515/aut-2017-0030 @ AUTEX

[28] A. Fabijańska, L. Jackowska-Strumiłło, Image processing and analysis algorithms for yarn hairiness determination, Mach. Vis. Appl. 23 (2012) 527-540.
[29] Z. Li, R. Pan, J. Zhang, B. Li, W. Gao, W. Bao. Measuring the unevenness of yarn apparent diameter from yarn sequence images, Meas. Sci. Technol. 27 (2016) 1-10. 